\title{
Correlación entre rendimiento, estabilidad fenotípica y métodos de selección simultánea en algodón (Gossypium hirsutum L.)
}

\section{Correlation among yield, phenotipic stability and simultaneus selection in cotton (Gossypium hirsutum L.)}

\author{
Carlos M. Sierra ${ }^{*}$, Miguel M. Espitia², Jorge Cadena ${ }^{3}$ \\ Recibido para publicación: Julio 27 de 2016 - Aceptado para publicación: Abril 18 de 2017
}

\begin{abstract}
RESUMEN
El rendimiento y la estabilidad fenotípica son necesarios para la liberación de nuevos cultivares. El objetivo de este estudio fue estimar la correlación lineal y de rango entre el rendimiento de fibra (RENFI), seis índices de estabilidad: $S^{2} \mathrm{i}$ (Roemer), $\sigma^{2}$ (Plaisted y Peterson), $b_{i}$ y $S_{d i}^{2}$ (Eberhart y Russell), $W_{i}$ (Wricke), $\sigma^{2}$, (Shukla), y cuatro índices de selección simultánea por RENFI y estabilidad: $P_{\mathrm{i}}$ (Lin y Binns), ER y SH (Eskridge) y $S_{i}^{(1)}$ (Huehn), en 10 genotipos (G) de algodón, evaluados en seis ambientes del Caribe Húmedo Colombiano. Se usó un diseño de bloques completo al azar por ambiente. El análisis de varianza combinado detectó diferencias altamente significativas para ambientes e interacción GxA. El RENFI no se correlacionó linealmente con los índices de estabilidad, en tanto que entre los índices de selección solo con $\mathrm{SH}$ presentó un nivel de asocio significativo $\left(r=0,65^{*}\right)$, siendo este inverso con respecto al resto de índices ( $r>-$ 0,43 ). Los seis índices de estabilidad se correlacionaron en forma positiva y significativa entre todos $\left(r \geq 0,71^{*}\right)$, menos $b_{i,}$ que sólo se correlacionó con $S^{2},\left(r=0,96^{* *}\right)$. El RENFI solo presentó correlación de rango $\left(r_{\mathrm{s}}=0,76^{* *}\right)$ con el $\mathrm{S}^{2}$. El índice $\sigma^{2}$ pa presentó correlación de rango significativa y positiva $\left(r_{\mathrm{s}}>0,68\right) \operatorname{con} \mathrm{S}^{2} \mathrm{~d}_{\mathrm{i}^{\prime}} \sigma^{2}{ }^{2}, \mathrm{~W}_{\mathrm{i}^{\prime}}$ ER y $\mathrm{SH}$. La clasificación de los genotipos por estabilidad fue igual y perfecta $\left(r_{\mathrm{s}}=1,0^{* *}\right)$ cuando se utilizó $\sigma^{2}{ }_{v^{\prime}} S^{2} d_{i^{\prime}}, \sigma^{2}{ }_{i}$ y $W_{i}$. La correlación de rango entre los índices de selección simultánea sólo fue similar $\left(r_{c}>0,82^{* *}\right)$ entre ER, $\mathrm{SH}$ y Pi. Los índices de selección simultánea ER y SH favorecieron la selección, por estabilidad, dada su $\mathrm{r}_{\mathrm{s}}>0,66$ significativa $\mathrm{y}$ directa con $\sigma_{\mathrm{va}^{\prime}}^{2} \mathrm{~S}^{2} \mathrm{~d}_{\mathrm{i}^{\prime}} \sigma_{\mathrm{i}}^{2}$ and $\mathrm{W}_{\mathrm{i}}$.
\end{abstract}

Palabras clave: Adaptabilidad, interacción genotipo $\mathrm{x}$ ambiente, métodos paramétricos y no paramétricos.

\begin{abstract}
Yield and phenotypic stability are necessary to release new cultivars. The aim of this study was to estimate the linear and rank correlation between fiber yield (RENFI), six stability indices: $S^{2}{ }_{i}$ (Roemer), $\sigma_{\text {va }}^{2}$ (Plaisted and Peterson), $b_{i}$ and $S^{2}{ }_{\text {di }}$ (Eberhart and Russell), Wa $W_{i}$ (Wricke), $\sigma^{2}{ }_{i}$ (Shukla), and four simultaneous selection indexes by RENFI and stability: $\mathrm{P}_{i}$ (Lin and Binns), ER and SH (Eskridge) and $\mathrm{S}_{i}^{(1)}$ (Huehn) in 10 cotton genotypes evaluated through six environments in humid Colombian Caribbean. A randomized complete block design was used for each environment. The combined variance analysis showed highly significant differences for environments and GXA interaction. RENFI did not correlate linearly with stability indices, whereas between selection indices only $\mathrm{SH}$ presented a significant association level $(r>-0.43)$, except SH $\left(r=0.65^{*}\right)$. The six stability indices showed positive and significant linear correlation between all $\left(r \geq 0.71^{*}\right)$ except $b_{i}$, which only was correlated with $\mathrm{S}^{2}\left(\mathrm{r}=0.96^{* *}\right)$. RENFI only showed rank correlation $\left(r_{\mathrm{s}}=0.76^{* *}\right)$ with $\mathrm{S}^{2}$. The $\sigma^{2}$ va index showed significant and positive rank correlation $\left(r_{\mathrm{s}}>0.68\right)$ with $\mathrm{S}^{2} \mathrm{~d}_{\mathrm{i}^{\prime}}$ $\sigma^{2}{ }^{\prime}, W_{i}, E R$ and $\mathrm{SH}$. The ranking for stability of genotypes was equal and perfect $\left(r_{\mathrm{s}}=1.0^{* *}\right)$ when used $\sigma^{2}{ }_{v^{\prime}} \mathrm{S}^{2} \mathrm{~d}_{\mathrm{i}^{\prime}}$ $\sigma^{2}$ and $W_{i}$. The rank correlation between simultaneous selection indices was only similar $\left(r_{s}>0.82 * *\right)$ between $\mathrm{ER}, \mathrm{SH}$ and $\mathrm{Pi}$. The simultaneous selection indices ER and $\mathrm{SH}$ favored selection, preferentially, for stability given its $r_{s}>0.66$ significant and direct with $\sigma^{2}{ }^{2} a^{\prime} S^{2} d_{i^{\prime}} \sigma_{i}^{2}$ and $W_{i^{\prime}}$.
\end{abstract}

Key words: Adaptability, Environment $x$ genotype interaction, Parametric and nonparametric methods.

\footnotetext{
${ }^{1 *}$ M. Sc. Investigador profesional CORPOICA. csierran@corpoica.org.co. Montería - Colombia

${ }^{2}$ Ph. D. Docente - investigador. Facultad de Ciencias Agrícolas - Universidad de Córdoba, Montería - Colombia. Carrera 6 No. $76-103$

- Fax: (4)7860255. Autor para correspondencia: mespitia37@ hotmail.com.

${ }^{3}$ Ph. D. Director CI. Turipaná, CORPOICA. Cereté - Colombia. jcadena@corpoica.org.co.
} 


\section{INTRODUCCIÓN}

El algodón es un cultivo de importancia socioeconómica en la región Caribe colombiana debido a que representa la principal actividad agrícola del segundo semestre del año y genera aproximadamente el $6 \%$ de los empleos directos e indirectos entre los cultivos transitorios que se siembran en el país. Durante la cosecha 2015/2016 en la región se sembraron 30.858 ha $(69 \%$ del área nacional) y se produjo el $55 \%$ de la fibra del país (24.712 t), ello representó un suministro de un poco más del $50 \%$ de la fibra y semilla para la industria textil y de aceites del país (Conalgodón 2015).

Debido a la alta variación en cuanto a suelos, clima y manejo agronómico entre localidades productoras, los cultivares expresan un comportamiento diferencial. Abbott y Pistorale (2011) atribuyen esta consecuencia a los efectos de la interacción genotipo $x$ ambiente (GxA). Sin embargo, mecanismos fisiológicos propios de cada cultivar como la adaptación y/o compensación también pueden explicar la manifestación de GxA (Karimizadeh et al. 2012; Dewdar 2013).

Entre las principales limitantes que la (GxA) plantea a un programa de mejora genética se destaca que, genera cambios en la clasificación o rango de los genotipos entre los ambientes, enmascara la expresión del carácter de interés, disminuye la correlación entre los valores fenotípicos y genotípicos, reducen el progreso de la selección y finalmente, dificulta la labor de selección y posterior recomendación de los genotipos para una o varias localidades (Kang y Pham 1991; Abate et al. 2015, Vargas et al. 2016). De esta forma, su análisis e interpretación son necesarios para definir una estrategia, bien sea, seleccionando genotipos con adaptación amplia o específica o identificando megaambientes, (González et al. 2007, 2010; Farshadfar et al. 2012, 2013).
Las metodologías implementadas para el estudio de los efectos de la interacción GxA son denominadas genéricamente como medidas de la estabilidad fenotípica (Kang y Pham 1991; González et al. 2010; Rea et al. 2015), las cuales difieren en los conceptos y en los procedimientos estadísticos adoptados para su estimación (Lin et al. 1986; Lin y Binns 1988; Abbott y Pistolare 2011; Espitia 2013). Los diferentes conceptos de estabilidad, conllevan al mejorador a preguntarse cuál estadístico de estabilidad debería ser usado para solucionar su problema particular (Liu et al. 2011).

A través del tiempo se han desarrollado numerosos métodos y modelos estadísticos para explicar la estabilidad y seleccionar cultivares superiores. Lin et al. (1986) describen tres conceptos o tipos de estabilidad agrupados en cuatro grupos. La estabilidad Tipo 1 (Grupo A), también Ilamada concepto estático (Léon 1986) o biológico de estabilidad (Becker 1981), considera un genotipo estable si su varianza a través de los ambientes es pequeña, estimadores como la varianza ambiental $S^{2} i$ (Roemer 1917) hacen parte de este grupo. La estabilidad Tipo 2 (Grupo B), denominada como el concepto dinámico (Léon 1986) o agronómico de estabilidad (Becker 1981), considera un genotipo estable si su respuesta a través de los ambientes es paralela a la respuesta media de todos los genotipos evaluados. Como ejemplos de este tipo mencionan a $\sigma^{2}{ }_{\text {va }}$ (Plaisted y Peterson 1959), $W_{i}\left(\right.$ Wricke 1962), y $\sigma^{2}{ }_{i}$ (Shukla 1972).

La estabilidad Tipo 3 considera que un genotipo es estable, si el cuadrado medio del error del modelo de la regresión sobre el índice ambiental es pequeño. Tiene en cuenta el comportamiento predecible e impredecible de un genotipo. Dentro de este tipo mencionan $a b_{i}$ (Eberhart y Russell 1966). Lin y Binns (1988) propusieron la estimación de la varianza del rendimiento de un cultivar $\left(\mathrm{P}_{\mathrm{i}}\right)$ con respecto al rendimiento promedio máximo dentro de la localidad, como el concepto de estabilidad Tipo 4, el cual es 
distinto respecto de los conceptos de estabilidad estático y dinámico (Mohammadi et al. 2012). En esencia, consideran que un genotipo es estable, si su varianza $\left(\mathrm{P}_{\mathrm{i}}\right)$ para un grupo de ambientes, es pequeña (Dehghani et al. 2008).

Numerosos métodos y modelos estadísticos paramétricos y no paramétricos, para realizar una selección de genotipos cada vez más precisa y refinada, se han propuestos a través del tiempo, varios autores los han relacionado, agrupado, comparado y correlacionado (González et al. 2010; Liu et al. 2011; Farshadfar et al. 2012; Karimizadeh et al. 2012; Sahin et al. 2012; Espitia 2013; Sabaghnia et al. 2013; Gedif et al. 2014; Vange et al. 2014; Sierra 2014; Abate et al. 2015; Vargas et al. 2016).

Lo anterior, demuestra el amplio uso y vigencia actual a nivel internacional, para explicar la interacción GxA y seleccionar los cultivares superiores a liberar comercialmente. No obstante, la amplia oferta de tales técnicas estadísticas, son pocos los trabajos que en Colombia se han reportado con el uso de la selección simultánea y la correlación de éstos con los parámetros de estabilidad fenotípica y el rendimiento (Espitia 2013).

La comparación de los índices que estiman la estabilidad junto con otros métodos de selección simultánea por alto rendimiento y estabilidad, permiten aumentar la oferta de herramientas estadísticas, mejoran el conocimiento y dominio de los mismos, además de facilitar su uso estadístico e interpretación adecuada, lo cual se reflejará en un proceso de liberación comercial de nuevos cultivares cada vez más refinado y eficiente, máxime cuando en el mundo y en Colombia, tradicionalmente los mejoradores de plantas han utilizado modalmente el método de Eberhart y Russell (1966), para cumplir tal propósito (Karimizadeh et al. 2012; Sahin et al. 2012; Espitia 2013; Sabaghnia et al. 2013; Farshadfar et al. 2013; Gomez et al. 2014; Gedif et al. 2014; Vange et al. 2014; Sierra 2014; Abate et al. 2015).

Teniendo en cuenta lo anterior y dado que el rendimiento de fibra (RENFI), la característica de interés económico más importante en el cultivo del algodón, es altamente influenciada por el ambiente; el presente estudio tuvo como objetivo fundamental, estimar la correlación lineal y de rango entre el RENFI, seis índices estabilidad: S2i (Roemer 1917), $\sigma_{\text {va }}^{2}$ (Plaisted y Peterson 1959), $b_{i}$ y $S^{2} d_{i}$ (Eberhart y Russell 1966), $W_{i}$ (Wricke 1962), $\sigma_{i}^{2}$ (Shukla 1972), y cuatro de índices de selección simultánea por RENFI y estabilidad: $P_{i}$ (Lin y Binns 1988), ER y SH (Eskridge 1990) y $S_{i}^{(1)}$ (Huehn 1990), en 10 genotipos de algodón evaluados en seis localidades del Caribe húmedo colombiano.

\section{MATERIALES Y MÉTODOS}

La investigación se llevó a cabo en seis ambientes representativos de la zona algodonera del Caribe húmedo colombiano, a saber: 1) Cereté 2008/2009 (C.I. Turipaná1); 2) Cereté 2008/2009 (El Cairo1); 3) Cereté 2009/2010 (C.I. Turipaná2); 4) Cereté 2009/2010 (El Cairo2); 5) Cereté 2009/2010 (El Principio); 6) Ciénaga de Oro 2009/2010 (Malagana). Como el número de experimentos no fue igual en todas las localidades, se considera aquí "ambiente" a la combinación de una localidad en un año determinado.

Se evaluaron 10 genotipos de algodón de fibra media, correspondientes a ocho líneas promisorias: LCER 0014, LCER 0016, LCER 0034, LCER 0044, LCER 0063, LCER 0060, LCER 0007, LCER 0046, obtenidas por el Centro de Investigación Turipaná de CORPOICA (Córdoba - Colombia) y dos variedades comerciales: SINUANA M - 137 (Nacional) y NuOpal (Foránea), utilizadas como testigos. Las líneas provienen del cruce de variedades altamente productivas y adoptadas por los agricultores de la región (Tabla 1). 
Tabla 1. Clasificación de 10 genotipos de algodón (Gossypium hirsutum L.) por rendimiento promedio de fibra (RENFI), seis índices de estabilidad fenotípica y cuatro de selección simultánea por RENFI y estabilidad.

\begin{tabular}{|c|c|c|c|c|c|c|c|c|c|c|c|c|c|c|c|c|c|c|c|c|}
\hline \multirow{3}{*}{$\frac{\text { GENOTIPO }}{\text { LCER } 0014}$} & \multirow{2}{*}{\multicolumn{2}{|c|}{$\begin{array}{l}\text { RENFI } \\
\text { kg ha }^{-1} \\
\end{array}$}} & \multirow{2}{*}{\multicolumn{2}{|c|}{$\begin{array}{c}\text { ROEMER } \\
\mathrm{S}^{2}{ }_{\mathrm{i}} \\
\end{array}$}} & \multirow{2}{*}{\multicolumn{2}{|c|}{$\begin{array}{c}\begin{array}{c}\text { PLAISTED\& } \\
\text { PETERSON }\end{array} \\
\sigma_{\text {va }}^{2} \\
\end{array}$}} & \multicolumn{4}{|c|}{ EBERHART\&RUSSELL } & \multirow{2}{*}{\multicolumn{2}{|c|}{$\begin{array}{c}\text { SHUKLA } \\
\sigma_{i}^{2} \\
\end{array}$}} & \multirow{2}{*}{\multicolumn{2}{|c|}{$\begin{array}{c}\text { WRICKE } \\
\mathrm{W}_{\mathrm{i}} \\
\end{array}$}} & \multicolumn{4}{|c|}{ ESKRIDGE } & \multirow{2}{*}{$\frac{\text { LIN\&BINNS }}{\mathrm{p}_{\mathrm{i}}}$} & \multirow{2}{*}{$\frac{\text { HUEHN }}{S_{i}^{(1)}}$} \\
\hline & & & & & & & $b_{i}$ & & $S^{2} d_{i}$ & & & & & & $b_{i}$ & & & ${ }^{2} d_{i}$ & & \\
\hline & 1107,5 & (5) & 397265 & (2) & 16181 & (6) & 10,8 & (8) 2 & 22790,72 & (2) & $27588,4^{*}$ & (6) & 503408 & (6) & 766 & (7) & 439 & (8) & $54928 \quad(8)$ & $3,0(5)$ \\
\hline LCER 0016 & 1149,1 & (2) & 506293 & (5) & 21622 & (9) & 0,8 & (6) 3 & $39232,54 * *$ & (5) & $39786,4^{* *}$ & (9) & 698576 & (9) & 756 & (8) & 465 & (6) & $47230 \quad(6)$ & $3,2(6,5)$ \\
\hline LCER 0034 & 1125,3 & (4) & 480696 & (4) & 18094 & (7) & 0,8 & (5) 3 & $31334,28 *$ & (4) & 31907,6 * & (7) & 572515 & (7) & 731 & (9) & 424 & (9) & $59358 \quad(9)$ & $3,7(8)$ \\
\hline LCER 0044 & 1098,0 & (7) & 944230 & (9) & 11529 & (5) & 1,3 & (9) & 4812,49 & (9) & 17135,6 & (5) & 336162 & (5) & 862 & (4) & 478 & (5) & 40634 & $3,2(6,5)$ \\
\hline LCER 0063 & 1043,5 & (10) & 1477646 & (10) & 62369 & (10) & 1,5 & (10) & $98971,59 * *$ & (10) & $131515^{* *}$ & (10) & 2166237 & (10) & 413 & (10) & 179 & (10) & 93451 (10) & 1,8 \\
\hline LCER 0060 & 1135,6 & (3) & 394106 & (1) & 8065 & (4) & 0,8 & (7) & 7971,56 & (1) & 9294,74 & (4) & 210709 & (4) & 893 & (3) & 507 & (3) & $41302 \quad$ (5) & $1,9(3)$ \\
\hline LCER 0007 & 1098,4 & (6) & 668038 & (7) & 4224 & (1) & 1,1 & (3) & 2750,4 & (7) & 673,39 & (1) & 72767 & (1) & 948 & (2) & 513 & (2) & 38138 & $1,4(1)$ \\
\hline LCER 0046 & 1062,6 & (9) & 530135 & (6) & 8044 & (3) & 1,0 & (2) & 12748,46 & (6) & 9291,63 & (3) & 210659 & (3) & 822 & (5) & 453 & (7) & $52949 \quad(7)$ & $2,8(4)$ \\
\hline SIN M-137 & 1229,2 & (1) & 446128 & (3) & 7925 & (2) & 0,9 & (4) & 10162,7 & (3) & 8989,06 & (2) & 205818 & (2) & 966 & (1) & 587 & (1) & $25804 \quad$ (1) & $3,9(9,5)$ \\
\hline NUOPAL & 1069,5 & (8) & 689638 & (8) & 18914 & (8) & 1,0 & (1) 3 & $37551,57^{* *}$ & (8) & $33784,5 *$ & (8) & 602544 & (8) & 788 & (6) & 494 & (4) & $35859 \quad$ (2) & $3,9(9,5)$ \\
\hline
\end{tabular}

Se empleó un diseño experimental de bloques completo al azar (DBCA) con diez tratamientos (genotipos) y cuatro repeticiones, el tamaño de parcela fue de seis surcos a 0,9 m entre surcos y $10 \mathrm{~m}$ de largo $\left(6 \times 0,9 \times 10=54 \mathrm{~m}^{2}\right)$. El análisis de varianza combinado previo, señaló diferencias altamente significativas para la interacción GxA (Sierra 2014).

Para lograr el objetivo del estudio, se utilizaron los datos del rendimiento de fibra (RENFI) obtenidos por el Centro de Investigación Turipaná de CORPOICA (Córdoba - Colombia), a través de la realización de seis pruebas de evaluación agronómica de 10 genotipos de algodón de fibra media en fincas de agricultores. Una vez detectada la significancia de la interacción GxA, se estimó la estabilidad fenotípica, a través de seis índices: $\mathrm{S}^{2}{ }_{\mathrm{i}}$ (Roemer 1917), $\sigma_{\text {va }}^{2}$ (Plaisted y Peterson 1959), $b_{i}$ y $S^{2} d_{i}$ (Eberhart y Russell 1966), $W_{i}$ (Wricke 1962), $\sigma^{2}{ }_{i}$ (Shukla 1972), y cuatro de índices de selección simultánea por RENFI y estabilidad: $\mathrm{P}_{i}$ (Lin y Binns 1988), ER y SH (Eskridge 1990) y $S_{i}^{(1)}$ (Huehn 1990).

Los cálculos para estimar los índices bi, $\mathrm{S}^{2} \mathrm{~d}_{\mathrm{i}^{\prime}}$ $\sigma^{2}{ }_{i} W_{i}$ ER y $\mathrm{SH}$ se realizaron según la rutina de acceso libre propuesta por Galindo (1992), para el software computacional SAS v9.3. Los parámetros $S^{2}{ }_{i} \sigma^{2}{ }_{v a} P_{i}$ y $S_{i}^{(1)}$ fueron calculados por medio del software de acceso libre GENES V.2013, 5.1 (Cruz 2013).
Para determinar el nivel de relación entre el RENFI, los seis índices de estabilidad y los cuatro índices de selección simultánea por RENFI y estabilidad, se realizó, un análisis paramétrico de correlación lineal simple de Pearson y un análisis no paramétrico de correlación de rango mediante la metodología propuesta por Spearman (Steel y Torrie 1980), usado como una medida de concordancia en la selección de los genotipos por RENFI, los índices de estabilidad y los de selección simultánea. Para ello las estimaciones obtenidas para los 10 genotipos estudiados, se clasificaron por orden de rango, siendo para el rendimiento y los índices de selección simultánea en forma decreciente (de mayor a menor) y para la estabilidad a la inversa (creciente).

\section{RESULTADOS Y DISCUSIÓN}

La tabla 1 muestra el rendimiento promedio de fibra (RENFI), los valores estimados de los seis índices de estabilidad fenotípica y los cuatro índices de selección simultánea para los 10 genotipos, además de la clasificación o el rango de los mismos entre paréntesis, según la preferencia en la selección de genotipos deseables. La clasificación varió de acuerdo a los valores de cada índice, lo cual se explica dado el concepto de estabilidad (Tipo 1, 2, 3 ó 4) que considera cada uno (Lin et al. 1986). Para el caso de los índices tenidos en cuenta en este estudio los genotipos deseables fueron aquellos que pre- 
sentaron menor valor de estimación, diferente de ER y SH, cuya consideración es la opuesta.

Ordinalmente LCER0060, LCER0014 y SIN-M-137, presentaron los menores valores de $\mathrm{S}^{2}$. Para este índice el genotipo deseable es aquel que no reacciona frente a condiciones cambiantes del ambiente (estabilidad tipo 1), por lo tanto prioriza la selección de genotipos con altos promedios de rendimiento.

La clasificación por $\sigma^{2}{ }_{\mathrm{va}}{ }^{\prime} \sigma^{2}{ }_{\mathrm{i}}$ y $\mathrm{W}_{\mathrm{i}}$ es consistente en indicar a LCER0007, SIN-M-137 y LCER0046 como los genotipos deseables. La varianza de estabilidad $\sigma^{2}{ }_{i}$ de Shukla (1972) destaca por su potencia para separar genotipos estables (Tipo 2) e inestables, además, al tratarse de una metodología multivariada aporta elementos precisos para la selección puesto que considera efectos aditivos (González et al. 2007, 2010; Gomez et al. 2014; Vange et al. 2014).

A pesar de que el $\mathrm{S}^{2} \mathrm{~d}_{\mathrm{i}}$ estima la estabilidad Tipo 3, coincide con $\sigma^{2}$ i en identificar como inestables a NUOPAL, LCER0063, LCER0034 y LCER0016. En tanto que el genotipo deseable fue LCER007, el cual presenta adaptación general sobre los ambientes evaluados $(b i=1)$. El Pi que estima estabilidad Tipo 4 clasificó como deseables los testigos SIN-M-137, NUOPAL y la línea LCER007.
En el proceso de selección de nuevos genotipos por estabilidad se deben tener en cuenta, principalmente, los conceptos de estabilidad Tipo 2 y Tipo 3, lo cual implica que los genotipos con esta característica, muestran alta estabilidad y responden positivamente a las mejoras tecnológicas en los procesos de producción, siendo esto de mucha importancia frente a los riesgos de cambio climático, contrario a la selección mediante el concepto de estabilidad Tipo 1.

Estos índices son de utilidad para evaluar la estabilidad de caracteres priorizados para ser mantenidos a cualquier costo en diferentes mega-ambientes, como lo sugieren varios estudios (Mahendra 2012; Espitia 2013; Sabaghnia et al. 2013; Gedif et al. 2014; Vange et al. 2014; Sierra 2014; Abate et al. 2015).

La tabla 2 muestra que el RENFI presentó correlación lineal negativa (inversa) con los índices de estabilidad y el índice de selección $\mathrm{P}_{\mathrm{i}}$. Estos coeficientes indican que a medida que el valor promedio del RENFI aumentó o disminuyó, en sentido contrario lo hacia el valor de estimación de los índices. A pesar de que el nivel de asociación no fue significativo $(r>-0,43)$, sugiere que es posible seleccionar genotipos sin sacrificar el rendimiento y la estabilidad, empleando indistintamente los índices mencionados, menos $\mathrm{ER}, \mathrm{SH}$ y $\mathrm{S}_{\mathrm{i}}^{(1)}$.

Tabla 2. Coeficientes de correlación lineal entre el rendimiento promedio de fibra (RENFI), seis índices de estabilidad fenotípica y cuatro de selección simultánea por rendimiento y estabilidad.

\begin{tabular}{|c|c|c|c|c|c|c|c|c|c|c|}
\hline \multirow{2}{*}{$\begin{array}{c}\text { MÉTODO } \\
\text { PARÁMETRO }\end{array}$} & \multirow{2}{*}{$\frac{\text { TRADICIONAL }}{\mathrm{S}^{2}{ }_{\mathrm{i}}}$} & \multirow{2}{*}{$\begin{array}{c}\begin{array}{c}\text { PLAISTED\& } \\
\text { PETERSON }\end{array} \\
\sigma_{\text {va }}^{2}\end{array}$} & \multicolumn{2}{|c|}{ EBERHART\&RUSSELL } & \multirow{2}{*}{$\frac{\text { SHUKLA }}{\sigma_{i}^{2}}$} & \multirow{2}{*}{$\frac{\text { WRICKE }}{\mathrm{W}_{\mathrm{i}}}$} & \multicolumn{2}{|c|}{ ESKRIDGE } & \multirow{2}{*}{$\frac{\text { LIN\&BINNS }}{\mathrm{p}_{\mathrm{i}}}$} & \multirow{2}{*}{$\frac{\text { HUEHN }}{S_{i}^{(1)}}$} \\
\hline & & & $b_{i}$ & $\mathbf{S}^{2} \mathbf{d}_{\mathrm{i}}$ & & & ER & $\mathrm{S}^{2} \mathrm{~d}_{\mathrm{i}}$ & & \\
\hline RENFI & $-0,58$ & $-0,45$ & $-0,57$ & $-0,43$ & $-0,45$ & $-0,45$ & 0,57 & $0,65 *$ & $-0,60$ & 0,39 \\
\hline $\mathrm{S}^{2}$ & & $0,80 * *$ & $0,96 * *$ & $0,71 *$ & $0,80 * *$ & $0,80 * *$ & $-0,71 *$ & $-0,78 * *$ & 0,66 * & $-0,36$ \\
\hline$\sigma^{2^{i}}$ & & & 0,59 & $0,98 * *$ & $1,00 * *$ & $1,00 * *$ & $-1,00 * *$ & $-0,90 * *$ & $0,87^{* *}$ & $-0,20$ \\
\hline$b^{\text {va }}$ & & & & 0,48 & 0,59 & 0,59 & $-0,49$ & $-0,60$ & 0,47 & $-0,38$ \\
\hline $\mathrm{S}^{2} \mathrm{~d}_{\mathrm{i}}$ & & & & & $0,98 * *$ & $0,98 * *$ & $-0,95 * *$ & $-0,89 * *$ & $0,83 * *$ & $-0,13$ \\
\hline$\sigma^{2}$ & & & & & & $1,00 * *$ & $-0,96 * *$ & $-0,93 * *$ & $0,87 * *$ & $-0,20$ \\
\hline$W_{i}$ & & & & & & & $-0,96 * *$ & $-0,93 * *$ & $0,87 * *$ & $-0,20$ \\
\hline$E R$ & & & & & & & & $1,00 * *$ & $-0,94 * *$ & 0,16 \\
\hline $\mathrm{SH}$ & & & & & & & & & $-0,98 * *$ & 0,37 \\
\hline$P_{\text {ig }}$ & & & & & & & & & & $-0,37$ \\
\hline
\end{tabular}

$S^{2}$ : Varianza a través de los ambientes de Roemer (1917); $\sigma^{2}$ : Varianza de la interacción GxA de Plaisted y Peterson (1959); b : Coeficiente de regresión y S² : Desvíos de la regresión de Eberhart y Russell (1966); $\sigma^{2}$ : Varianza de la estabilidad de Shukla (1972); W: Ecovalencia de Wricke (1965); ER: Cota de Eberhart y Russell y SH: cota de Shukla para Eskridge (1990); $P_{i}$ : Medida de superioridad de un cultivar de Lin y Binns (1988); $S_{i}^{(1)}$ : Índice no paramétrico de Huehn (1990); * y ** Coeficiente de correlación líneal significativos al 5\% y $1 \%$, respectivamente para la prueba $\mathrm{t}$. 
Los seis índices de estabilidad presentaron correlación lineal positiva y significativa entre todos, excepto bi, $\left(r>-0,71^{*}\right)$ que sólo mostró correlación con $\mathrm{S}^{2}{ }_{i} \quad\left(\mathrm{r}=0,96^{* *}\right)$. La correlación fue directa y altamente significativa $\left(r>0,98^{* *}\right)$ entre los índices $\sigma^{2}{ }_{v a} S^{2} d_{i}, \sigma^{2}{ }_{i}$ y $W_{i}$. Asociaciones similares entre los estimadores de estabilidad han sido reportadas por otros autores en diferentes especies cultivadas (Espitia et al. 1993; Vertel et al. 1999; Espitia et al. 2003; González et al. 2010; Liu et al. 2011; Karimizadeh et al. 2012; Mahendra, 2012; Farshadfar et al. 2013; Tadege et al. 2014).

Solo SH presentó correlación positiva y significativa $\left(r=0,65^{*}\right)$ respecto del RENFI. Lo cual sugiere que la selección basada en este índice consideró en forma directa al RENFI, así como también la estabilidad. Esto es muy importante en el proceso de selección y liberación comercial de nuevos cultivares, ya que son deseables genotipos que expresen simultáneamente ambos atributos. El nivel de asocio entre los índices de selección simultánea ER y SH fue completo y directo $\left(r=1,00^{* *}\right)$, mientras que entre estos con $\mathrm{Pi}$, la relación es significativa, pero inversa $\left(r>-0,94^{* *}\right)$.

El índice de selección $\mathrm{S}_{\mathrm{i}}^{(1)}$ no estuvo asociado con el RENFI, los seis índices de estabilidad, como tampoco con los tres índices de selección simultánea. Estos resultados concuerdan a los reportados en algodón y otras especies cultivadas por (León 1986; Kang y Pham 1991; Espitia et al. 1993; Espitia et al. 2003; Mahommadi et al. 2009; Farshadfar et al. 2012; Tadege et al. 2014; Gedif et al. 2014). Como se hacía mención anteriormente, es importante la escogencia del método para la selección de genotipos a recomendar.

La tabla 3 muestra la correlación de rango entre el RENFI, los índices de estabilidad y de selección simultánea. Donde se aprecia la significancia entre el RENFI y $\mathrm{S}^{2}{ }_{\mathrm{i}}\left(\mathrm{r}_{\mathrm{s}}=0,76^{* *}\right)$, indicando que ambos parámetros clasificaron a los genotipos en forma similar, corroborando lo argumentado de la tabla 1. El índice de selección $\mathrm{S}_{i}^{(1)}$ emplea el mismo concepto de estabilidad (Tipo 1) que $S_{i}^{2}$, sin embargo, presentó correlación negativa $\left(r_{s}=-0,43\right)$.

La clasificación de los genotipos por estabilidad fue igual $\left(r_{\mathrm{s}}=1,00^{* *}\right)$ para $\sigma^{2}{ }_{{ }^{\prime} a^{\prime}} \sigma^{2}{ }_{\mathrm{i}} \mathrm{y} \mathrm{W}_{\mathrm{i}^{\prime}}$ pero varió respecto de la realizada por $S^{2} d_{i}\left(r_{s}=0,89 * *\right)$ y los índices de selección simultánea ER y SH $\left(r_{s}>0,68\right)$. Estas asociaciones son consistente con los resultados de las Tablas 1 y 2. La similitud en la clasificación de los genotipos cuando se emplearon los índices $\sigma^{2}{ }_{v a} \sigma^{2}{ }_{i}$ y $W_{i^{\prime}}$ obedece a que utilizan el mismo concepto de estabilidad (Tipo 2), de acuerdo a como lo explican varios

Tabla 3. Coeficientes de correlación de rangos entre el rendimiento promedio de fibra (RENFI), seis índices de estabilidad fenotípica y cuatro de selección simultánea por rendimiento y estabilidad.

\begin{tabular}{|c|c|c|c|c|c|c|c|c|c|c|}
\hline \multirow{2}{*}{$\begin{array}{c}\text { MÉTODO } \\
\text { PARÁMETRO }\end{array}$} & \multirow{2}{*}{$\begin{array}{c}\text { TRADICIONAL } \\
\mathrm{S}_{\mathrm{i}}^{2}\end{array}$} & \multirow{2}{*}{ 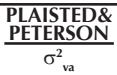 } & \multicolumn{2}{|c|}{ EBERHART\&RUSSELL } & \multirow{2}{*}{$\frac{\text { SHUKLA }}{\sigma^{2}{ }_{i}}$} & \multirow{2}{*}{$\frac{\text { WRICKE }}{\mathrm{W}_{\mathrm{i}}}$} & \multicolumn{2}{|c|}{ ESKRIDGE } & \multirow{2}{*}{$\frac{\text { LIN\&BINNS }}{\mathrm{p}_{\mathrm{i}}}$} & \multirow{2}{*}{$\frac{\text { HUEHN }}{S_{i}^{(1)}}$} \\
\hline & & & $b_{i}$ & $S^{2} d_{i}$ & & & ER & $S^{2} d_{i}$ & & \\
\hline RENFI & $0,76^{* *}$ & 0,24 & 0,02 & $-0,19$ & $-0,24$ & 0,24 & 0,33 & 0,45 & 0,32 & $-0,43$ \\
\hline $\mathrm{S}^{2}$ & & 0,32 & 0,05 & 0,20 & 0,32 & 0,32 & 0,26 & 0,22 & 0,03 & $-0,54$ \\
\hline$\sigma_{\text {va }}^{i}$ & & & 0,39 & 0,89 ** & $1,00 * *$ & $1,00^{* *}$ & $0,89 * *$ & $0,68 *$ & 0,54 & 0,37 \\
\hline$b_{i}$ & & & & 0,13 & 0,39 & 0,39 & 0,36 & 0,44 & 0,52 & $-0,03$ \\
\hline $\mathrm{S}^{2} \mathrm{~d}_{\mathrm{i}}$ & & & & & $0,89 * *$ & $0,89 * *$ & $0,85 * *$ & $0,66^{*}$ & 0,53 & 0,43 \\
\hline$\sigma_{i}^{2}$ & & & & & & $1,00 * *$ & $0,89 * *$ & 0,68 * & 0,54 & 0,37 \\
\hline$W_{i}$ & & & & & & & $0,89 * *$ & $0,68 *$ & 0,54 & 0,37 \\
\hline ER & & & & & & & & $0,92 * *$ & $0,82 * *$ & 0,43 \\
\hline $\mathrm{SH}$ & & & & & & & & & $0,94 * *$ & 0,43 \\
\hline$P_{\text {ig }}$ & & & & & & & & & & 0,43 \\
\hline
\end{tabular}

$\mathrm{S}^{2}$ : Varianza a través de los ambientes de Roemer (1917); $\sigma^{2}{ }_{\mathrm{va}}$ : Varianza de la interacción GxA de Plaisted y Peterson (1959); b: Coeficiente de regresión y S² $\mathrm{d}_{\mathrm{i}}$ : Desvíos de la regresión

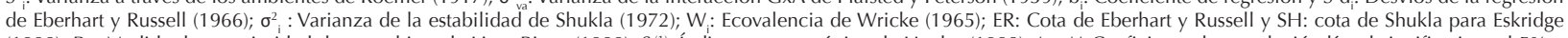
(1990); $P_{i}$ : Medida de superioridad de un cultivar de Lin y Binns (1988); $S_{i}^{(1)}$ : Índice no paramétrico de Huehn (1990); * y ** Coeficiente de correlación líneal significativos al 5\% Y $1 \%$, respectivamente para la prueba $\mathrm{t}$. 
autores (Lin et al. 1986; Vertel et al. 1999; González et al. 2010; Farshadfar et al. 2012, 2013; Karimizadeh et al. 2012; Tadege et al. 2014; Gedif et al. 2014). No obstante, a pesar de que algunos índices estiman la estabilidad fenotípica mediante conceptos diferentes, la clasificación o asignación de rangos a los genotipos algunas veces puede ser similar, como lo reporta Sabaghnia et al. (2013).

A correlación de rango entre los índices de selección simultánea fue similar $\left(r_{s}>0,82 * *\right)$ entre $E R, S H$ y $P_{i}$, pero estadísticamente no significativa con $\mathrm{S}_{i}^{(1)}$. La mayor concordancia en la clasificación de genotipos se presenta entre los índices ER y SH $\left(0,92^{* *}\right)$ y los índices $\mathrm{SH}_{\text {y }} \mathrm{P}_{\mathrm{i}}$ $\left(0,94^{* *}\right)$. La clasificación de genotipos deseables por los índices de selección simultánea ER y $\mathrm{SH}$ estuvo influenciada preferencialmente por la estabilidad, dada su correlación de rango significativa y directa $\left(r_{\mathrm{s}}>0,66\right)$ con $\sigma^{2}{ }_{v^{\prime}} \mathrm{S}^{2} \mathrm{~d}_{\mathrm{i}^{\prime}}$ $\sigma^{2}{ }_{i}$ y $W_{i}$. Es de señalar que aunque el índice de superioridad $\mathrm{Pi}$ estima estabilidad Tipo 4, en este estudio no mostró correlación lineal y/o de rango con ninguno de los índices de estabilidad considerados o con el RENFI. No obstante, ofrece una estrategia adicional para la selección simultánea y caracterización de los patrones de respuesta de los genotipos a los cambios ambientales, la cual junto con otros permite la extrapolación a una gama mucho más amplia de entornos de aquellos ya evaluados (Sahin et al. 2012). En este estudio, el índice de selección $\mathrm{Si}^{(1)}$ no mostró correlación de rango significativa con el RENFI, los seis índices de estabilidad y tampoco con los otros tres índices de selección simultánea, sugiriendo que es un método que involucra estimaciones y conceptos diferentes de selección al de sus similares. Estos resultados son similares a los reportados en algodón y otras especies cultivadas (León, 1986; Kang y Pham 1991; Espitia et al. 1993; Espitia et al. 2003; Mahommadi et al. 2009; Farshadfar et al. 2012, 2013; Mohammadi et al. 2012; Tadege et al. 2014; Gedif et al. 2014).

\section{CONCLUSIONES}

El rendimiento de fibra de los 10 genotipos no mostró correlación lineal significativa con los seis índices de estabilidad y los cuatro de selección simultánea ( $r>-0,43)$, a excepción del índice $\mathrm{SH}\left(\mathrm{r}=0,65^{*}\right)$.

Los seis índices de estabilidad presentaron correlación lineal positiva y significativa entre todos $\left(r \geq 0,71^{*}\right)$, excepto $b_{i}$, que sólo mostró correlación con $\mathrm{S}^{2}{ }_{\mathrm{i}}\left(\mathrm{r}=0,96^{* *}\right)$.

EI RENFI sólo mostró correlación de rango positiva y significativa $\left(r_{s}=0,76^{* *}\right)$ con el $\mathrm{S}^{2}$. El índice $\sigma_{\text {va }}^{2}$ presentó correlación de rango positiva $\left(r_{s}>0,68\right)$ con $S^{2} d_{i^{\prime}} \sigma^{2}{ }_{i}, W_{i^{\prime}}$ ER y $S H$.

La clasificación por estabilidad de los genotipos fue igual y perfecta $\left(r_{s}=1,0^{* *}\right)$ cuando se utilizó $\sigma^{2}{ }_{v a^{\prime}} \sigma^{2}{ }_{i}$ y $W_{i^{\prime}}$ por lo tanto podrían ser utilizados indistintamente para la identificación de genotipos estables.

La correlación de rango entre los índices de selección simultánea sólo fue similar $\left(r_{s}>0,82 * *\right)$ entre $\mathrm{ER}, \mathrm{SH}$ y $\mathrm{P}_{\mathrm{i}}$. Los índices de selección simultánea ER y SH favorecieron la selección preferencialmente por estabilidad, dada su $r_{\mathrm{s}}>0,66$ significativa y directa con $\sigma_{{ }^{2} a^{\prime}}, S^{2} d_{i^{\prime}}, \sigma^{2}{ }_{i}$ y $W_{i}$, no obstante los tres índices clasifican los genotipos de forma similar y permitieron la selección simultánea de algunos genotipos, que combinaron altos rendimiento y estabilidad, lo cual es muy deseable en la agricultura actual para afrontar los desafíos que impone el calentamiento global y cambio climático.

\section{REFERENCIAS}

Abate, F., Mekbib, F. and Dessalegn, Y. 2015. Association of Different Parametric and Non parametric Stability Models in Durum Wheat (Triticum turgidum Desf.) Genotypes. International Journal of Plant \& Soil Science. 7(4):192-201. 
Abbott, L. and Pistorale, S. 2011. Análisis de la estabilidad y adaptabilidad de caracteres de interés agronómico en genotipos selectos de cebadilla criolla (Bromus catharticus). Agriscientia. 28(2):109-117.

Becker, H.C. 1981. Correlations among some statistical measures of phenotypic stability. Euphytica 30: 835-840.

Conalgodón, Confederación Colombiana del Algodón. 2015. InformeCosecha Nacional - Julio 2015. http://conalgodon.com/ wp-content/uploads/2015/12/201507Informe-Cosecha-Nacional.pdf (20 jun 2016).

Cruz, C. 2013. Programa Genes V. 2013, 5.1. Aplicativo computacional em genética e estatística. http://www.ufv.br/dbg/genes/ genes.htm [22 sep 2013].

Dewdar, M. 2013. Stability analysis and genotype $x$ environment interactions of some Egyptian cotton cultivars cultivated. African Journal of Agricultural Research. 8(41):5156-5160.

Dehghani, H., Sabaghpour, S. and Sabaghnia, N. 2008. Genotype $x$ environment interaction for grain yield of some lentil genotypes and relationship among univariate stability statistics. Spanish J. Agric. Res. 6(1):385-394.

Eberhart, A. and Russel, W. 1966. Stability parameters for comparing varietes. Crop. Sci. 6(1):36-40.

Eskridge, K. 1990. Selection of stable cultivars using a safety-first rule. Crop. Sci. 30(2):369-374.

Espitia, M. 2013. Importancia dela adaptabilidad y estabilidad fenotípica en la liberación comercial de nuevas variedades de fríjol caupí. En Primera Jornada Tecnológica Internacional Sobre Fríjol Caupí. Memorias en CD. Montería, Universidad de Córdoba, noviembre/28-29/2013.
Espitia, M., Araméndiz, H. y Arrieta, P. 2003. Selección simultanea por altos rendimientos y estabilidad de genotipos de frijol caupí (Vigna unguiculata L.). Rev. Fitotecnia Colombiana. 3(1):17-22.

Espitia, M., Araméndiz, H. y Mendoza, A. 1993. Selección simultánea de genotipos de algodón por altos rendimiento y estabilidad. Revista ICA. 28(1):227-234.

Farshadfar, E., Sabaghpour, S. and Zali, H. 2012. Comparison of parametric and nonparametric stability statistics for selecting stable chickpea (Cicer arietinum L.) genotypes under diverse environments. Australian Journal of Crops Science. 6(3):514-524.

Farshadfar, E., Mahtabi, E., Mahdi, and M. 2013. Assessment of parametric stability statistics for selecting stable Chickpea genotypes. International Journal of Agriculture and Crop Sciences. 5(21):2568-2575.

Galindo, J. 1992. Análisis de estabilidad fenotípica mediante el sistema SAS. Revista ICA. 27(1):4961-4962.

Gedif, M., Yigzaw, D. and Tsige, G. 2014. Genotype-environment interaction and correlation of some stability parameters of total starch yield in potato in Amhara region, Ethiopia. Journal of Plant Breeding and Crop Science. 6(2):31-40.

Gomez, G., Uneda-Trevisoli, S., Pinheiro, J. and Di Mauro, A. 2014. Adaptive and agronomic performances of soybean genotypes derived from different genealogies through the use of several analytical strategies. African Journal Agricultural Research. 9(28):2146-2157.

González, T., Monteverde, E., Marín, C. y Madríz, P. 2007. Comparación de tres métodos para estimar estabilidad del rendimiento en nueve variedades de algodón. Interciencia. 32(5):334-348. 
González, A., Pérez, D., Sahagún, J., Franco, O., Morales, E., Rubí, M., Gutiérrez, F. y Balbuena, A. 2010. Aplicación y comparación de métodos univariados para evaluar la estabilidad en maíces del valle Toluca Atlacomulco, México. Agronomía Costarricense. 34(2):129-143.

Huehn, M. 1990. Nonparametric measures of phenotypic stability. Part 1: Theory, Euphytica. 47(1):189-194.

Kang, M. and Pham, H. 1991. Simultaneous selection for high yielding and stable crop genotypes. Agron. Journal. 83(1): 161-165.

Karimizadeh, R., Mohammadi, M., Sabaghnia, N., Shefazadeh, M. and Pouralhossini, J. 2012. Univariate stability analysis methods for determining genotype $x$ environment interaction of durum wheat grain yield. African J. Biotech. 11(1):2563-2573.

León, J. 1986. Methods of simultaneous estimation of yield and yield stability. In: Biometrics in Plant Breeding. Proc. 6th Meeting. Eucarpia, section Biometrics in Plant Breeding, Birmingham: 299-308.

Lin, C., Binns, M., and Lefkovitch, L. 1986. Stability analysis: where do we stand? Crop Sci. 26(1):894-900.

Lin, C. and Binns, M. 1988. A method of analyzing cultivar $\mathrm{x}$ location $\mathrm{x}$ year experiments: a new stability parameter. Theor. Appl. Gene. 76(3):425-430.

Liu, Y., Hu, W., Yuan-qi, W., and Yu-bi, H. 2011. Yield Stability of Maize Hybrids Evaluated in National Maize Cultivar Regional Trials in Southwestern China Using Parametric Methods. Agricultural Sciences in China. 10(9):1323-1335.
Mahendra, D. 2012. Genotype x Environment Interaction and Stability analysis of Performance in Watermelon. Partial fulfillment of the requirements for the degree of Doctor of Philosophy Horticultural Science Raleigh, North Carolina State University, North Carolina, 94p.

Mahommadi, R., Aghaee, M., Haghparast, R., Pourdad, S., Rostaii, M., Ansari, Y., Abdolahi, A. and Amri, A. 2009. Association among non-parametric measures of phenotypic stability in four annual crops. Middle Eastern and Russian Journal of Plant Science and Biotechnology. Special issue. 3(1):20-24.

Mohammadi, M.; Karimizadeh, R.; Sabaghnia, N.; Shefazadeh, K. 2012. Genotype environment interaction and yield stability analysis of new improved bread wheat genotypes, Turkish Journal of Field Crops, 2012, 17(1): 67-73.

Plaisted, R. and Peterson, L. 1959. A technique for evaluating the ability of selections to yield consistence in different locations or seasons. American Potato Journal. 36(1):381-385.

Rea, R., De Sousa-Vieira, O., Díaz, A., Ramón, M., Briceño, R., George, J. and Demey, J. 2015. Assessment of yield stability in sugarcane genotypes using non-parametric methods. Agronomía Colombiana. 33(2):131-138.

Roemer, J. 1917. Sinde die ertagdreichen Sorten ertagissicherer. Mitt DLG, 32(1):87-89.

Sabaghnia, N., Mohammadi, M. and Karimizadeh, R. 2013. Yield stability of performance in multi environment trials of barley (Hordeum vulgar L.) genotypes. Acta Universitatis Agriculturae et Silviculturae Mendeliane Brunensis. 61(3):787-793. 
Sahin, E., Zeinalzadeh, T. and Tosun, M. 2012. Genotype by environment interaction and stability analysis of orchardgrass (Dactylis glomerata L.) ecotypes for seed yield in Erzurum, Turkey. International Journal of Agriculture and Crops Sciences. 4(2):45-50.

Shukla, 1972. Some statistical aspects of partitioning genotype - enviromental components of variability. Heredity. 29(1):237-245.

Sierra, C. 2014. Análisis de estabilidad fenotípica y genealógico para la selección de líneas promisorias de algodón (Gossypium hirsutum L.) para el Caribe Húmedo. Trabajo de grado de maestría en Ciencias Agronómicas, área de formación en Fitomejoramiento. Facultad de Ciencias Agrícolas. Universidad de Córdoba. 189p.

Steel, M. and Torrie, J. 1980. Principles and procedures of statistics. McGraw-Hill. New York, United States. 633p.
Tadege, M., Utta, H. and Aga, A. 2014. Association of statistical methods used to explore genotypic $\mathrm{x}$ environment interaction (GEI) and cultivar stability. African Journal Agricultural Research. 9(29):2231-2237.

Vange, T., Ango, I. and Adedzwa, D. 2014. Stability Analysis of Six Improved Sorghum Genotypes across Four Environments in the Southern Guinea Savanna Agroecological Zone of Nigeria. International Journal of Advances in Agricultural Science and Technology. 2(2):01-14.

Vargas, E., Vargas, J. and Baena, D. 2016. Análisis de estabilidad y adaptabilidad de híbridos de maíz de alta calidad proteica en diferentes zonas Agroecológicas de Colombia. Acta Agron. 65(1):72-79.

Vertel, M., Espitia, M. y Martinez, R. 1999. Comparación de ocho índices para determinar estabilidad fenotípica en algodón (Gossypium hirsutum L). Agronomía Colombiana. 16(1-3):30-34.

Wricke, G. 1962. On a method of understanding the biological diversity in field research.Z. Pflanzenzucht. 47(1):92-96. 